\title{
Functional community assembly and turnover along elevation and latitude
}

$5{ }^{1}$ Department of Evolution, Ecology and Organismal Biology, The Ohio State University

318 W. 12th Avenue, 300 Aronoff Laboratory, Columbus OH, 43210

$7 \quad$ 2Department of Ecology and Evolutionary Biology, Yale University

8165 Prospect Street, New Haven, CT, 06520, USA

$9{ }^{3}$ Institut de Biologie de l'ENS (IBENS), Département de biologie, École normale supérieure, CNRS,

10 INSERM, Université PSL, 75005 Paris, France

11

12 Email addresses: Marta A. Jarzyna: jarzyna.1@osu.edu

16 Keywords: biodiversity, birds, community assembly, elevational gradient, functional diversity, latitudinal

17 gradient, traits

18

19 Corresponding author: Marta A. Jarzyna, Department of Evolution, Ecology and Organismal Biology, The

20 Ohio State University, 318 W. 12th Avenue, 300 Aronoff Laboratory, Columbus OH, 43210. 


\section{Abstract}

24 The drivers of community coexistence are known to vary with environment, but their consistency across

25 latitudes and scales, and resulting conservation implications, remain little understood. Here, we

26 combine functional and phylogenetic evidence along elevations to document strong biotic constraints

27 on coexistence in avian communities in both benign (tropical low elevations) and severely harsh

28 (temperate/polar highlands) environments. Assemblages in both are marked by high assemblage

29 functional uniqueness, whereas in tropical highlands and temperate/polar low elevations there is strong

30 functionally redundancy and pronounced environmental constraints. Only in harsh environments is

31 phylogeny an effective surrogate for functional assemblage structure, reflecting nuanced shifts in the

32 position, shape, and composition of measured multivariate trait space along gradients. Independent of

33 scale and latitude, high elevation assemblages emerge as exceptionally susceptible to functional change.

\section{Introduction}

36 Species, and the communities they form, convey a range of functions to ecosystems and humans that

37 are now under increasing threat from global change (1). Drivers, presumed mechanisms, and evidence

38 of community change are non-uniform among latitudes and elevations (2-4). This raises fundamental

39 questions about the processes and relative roles of biotic and abiotic drivers underpinning community

40 coexistence along large-scale spatial and environmental gradients and their implications for the

41 maintenance of community functions (5-8). While biotic constraints such as competitive constraints on

42 trait equivalencies, i.e., limiting similarity (9), or certain facilitative intercations involving unique species

43 (10-12) are expected to increase divergence (i.e., overdispersion) of trait characteristics among

44 community members, particularly for closely related species, environmental constraints (or filters) that

45 select for common phenotypes might decrease trait divergence and enhance trait similarity (i.e.,

46 clustering) (13). The latter are expected to dominate toward harsh and less stable environments (13, 
47 14), whereas biotic constraints, particularly competitive exclusion, should be more prominent in

48 productive and stable settings $(15,16)$. Despite their key role for gauging the functional consequences of

49 climate change, whether and how these processes and resulting patterns hold from local to

50 regional/global scales, where macroevolutionary constraints and contingencies on clade functional

51 space emerge, remains largely unclear (17-19) or reliant on phylogenetic proxies of uncertain surrogacy

52 value (20-22).

54 Here, we use the natural experiment provided by elevational gradients of the world's main mountain

55 regions replicated along latitude $(23,24)$, a time-calibrated phylogeny $(25)$, comprehensive trait data

56 (26), and elevational distributions (24) addressing nearly all extant bird species, to test how functional

57 structure varies from benign to harsh conditions across local, regional, and global scales. For 8,410

58 assemblages along elevations from sea level to 7,340 meters, we estimate prevalence of assembly

59 mechanisms using dendrogram-based functional diversity (FD), hypervolume-based functional diversity

$60 \quad\left(\mathrm{FD}_{\mathrm{H}}\right.$; Supplementary Material), and species' local functional distinctness (FDI) which captures the

61 distinct contribution species make to the total functional diversity of a local assemblage (27). We use

62 their species richness-controlled values, cFD and cFDI (standardized effect sizes), supported by quantile

63 scores and associated p-values, to distinguish overdispersion from clustering. To investigate the

64 evolutionary underpinnings of species coexistence and perform a widely called for test of phylogenetic

65 and functional structure surrogacy $(20,22,28)$ we use comprehensive phylogenetic information for all

66 assemblages. Finally, we assess the shape, position, and turnover in assemblage's multivariate trait

67 volume and decompose it into its constituent elements (single traits) to gain a more mechanistic

68 understanding of assemblage functional structure $(29,30)$.

69 


\section{$71 \quad$ Results}

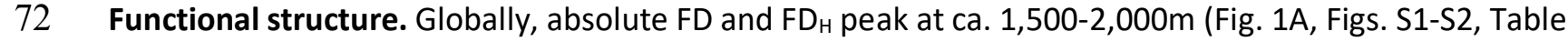

73 S1). Both low and high elevations exhibit functional overdispersion with higher levels of FD and mean

74 FDI ( $\left(\mathrm{FDI}_{\text {avg }}\right)$ than their species richness would suggest (cFD and cFDI patterns, Fig. 1B,E). In contrast, mid

75 elevations are generally more functionally clustered (Fig. 1B,E). But a strong latitudinal variation in this

76 pattern is evident (Fig. 1, Figs. S1-S3, Table S1). cFD, $\mathrm{CFD}_{\mathrm{H}}$, and $\mathrm{CFDl}_{\text {avg }}$ decline along elevation in the

77 tropics $\left(<23.5^{\circ}\right)$ and sub-tropics $\left(23.5-35^{\circ}\right)$, but increase toward high elevations in temperate and polar

78 regions ( $>35^{\circ}$; Fig. 1B,E, Figs. S1-S2, Table S1). Accordingly, cFD, cFD ${ }_{H}$, and cFDI $_{\text {avg }}$ decrease with latitude

79 in low to mid elevations, but this trend reverses for high elevations (Fig. 1C,F, Figs. S1-S2, Table S1).

80 Species' local functional role is highly uneven along elevation and latitude, with a small number of

81 species contributing much more functional uniqueness than the rest in regions with strong

82 environmental constraints (Fig. 1G-I, Figs. S1-S2, Table S1). While the species richness-controlled

83 skewness of species FDI values, $\mathrm{CFDI}_{\text {skew, }}$ increases toward higher elevations in the tropics and sub-

84 tropics, the opposite applies to temperate and polar regions (Fig. 1H, Figs. S1-S2, Table S1).

85 Consequently, cFDI skew increases from the tropics to the arctic at low to mid elevations (Fig. 1I, Figs. S1-

86 S2, Table S1), but declines at high elevations.

88 Phylogenetic and functional structure surrogacy. Absolute (PD) and richness-controlled (cPD)

89 phylogenetic diversity follow elevational and latitudinal trends broadly similar to those of function (Fig.

90 1J,K,L, Fig. S1, Table S1). The phylogenetic structure of an assemblage provides a reasonable proxy for

91 the functional structure, but in a strongly latitude-dependent way (Fig. 2C,D, Table S2). In the tropics,

92 the phylogenetic structure offers reasonable, but not strong, surrogacy for functionally overdispersed

93 (frequency, $\mathrm{Fr}$, with which $\mathrm{cPD}>0$ sites are also $\mathrm{cFD}>0$; $\mathrm{Fr}=0.63$ ) and clustered (frequency with which

$94 \quad \mathrm{CPD}<0$ sites are also $\mathrm{CFD}<0 ; \mathrm{Fr}=0.62$ ) assemblages (Fig. 2D, Table S2). Toward higher latitudes, the 
95 predictive power of phylogenetic structure diverges strongly (Fig. 2D, Table S2), reaching respectively

$96 \mathrm{Fr}=0.21$ and $\mathrm{Fr}=0.81$ for functionally overdispersed and clustered assemblages in temperate and polar

97 regions. This general latitudinal pattern of the predictive ability of the phylogeny is upheld by quantile

98 scores and associated estimated p-values (two-tailed test, $\alpha=0.05$; Fig S5, Table S2).

99

100 Turnover and decomposition of the multivariate trait volume. Globally, the volume of assemblage

101 traits and its relative (centroid) location vary little up to ca. 3,500m elevation, but both shift drastically

102 above that (Fig. 3A,B), suggesting strong across elevation assemblage trait volume redundancy from low

103 to mid elevations and increasing uniqueness above. This is confirmed by the global pattern of overlap in

104 assemblage trait volume (as Sørensen similarity), FDo, whose rapid decline toward high elevations (Fig.

$1053 \mathrm{3}$ ) coincides with the global peak in functional clustering (at ca. 2,000m; Fig. 1). This global pattern

106 mirrors those in tropical mountain systems, but not those in temperate and polar regions where FD。

107 slightly increases toward higher elevations (Fig. 3C). Simpson's similarity, FD, remains high and

108 relatively constant along elevation both globally and in the tropics, but increases slightly in higher

109 latitude regions (Fig. 3D). Fraction of trait volume unique to lower elevation assemblages (FDuI)

110 increases strongly toward higher elevations globally and in the tropics, but this increase is less

111 pronounced for temperate and polar regions (Fig. 3E). Fraction of trait volume unique to higher

112 elevation assemblages (FDuh) declines slightly along the elevational gradient globally and for high

113 latitude regions, but shows a slight humped pattern in the tropics (Fig. 3F).

115 The variation in avian trait volume along elevation and latitude we uncover is underpinned by a strongly

116 expected (Fig. S6) change in the prevalence of select traits (Fig. 4, Figs. S7,S8). Certain functional

117 components-e.g., mid- and upper-canopy and open-water foragers-generally decline drastically with

118 increasing elevation (Fig. 4B, Figs. S7,S8). Nocturnal species also disappear toward higher elevations (Fig. 
$1194 \mathrm{E})$, which we attribute to harsher night-time temperatures and decreased daytime competition. The

120 prevalence of birds of prey, granivorous, and ground foraging birds increases steeply linearly with

121 elevation in temperate and arctic regions, but closer to the equator shows a mid-elevation peak and a

122 steep decline thereafter (Fig. 4D, Figs. S7,S8). The proportion of insectivorous species increases with

123 elevation in the tropics, but not in temperate and arctic regions, suggestive of lower insect abundance at

124 high elevations there (Fig. 4C). Average assemblage body mass declines from lower to mid elevations

125 and then, at least in higher latitudes, strongly increases toward high elevations (Fig. 4F), consistent with

126 Bergmann's rule type pattern (31).

127

128 Discussion

129 Our results confirm our expectation around a prominence of biotic interactions (as suggested by

130 overdispersion) in more productive and stable environments (i.e., tropical low elevations).

131 Correspondingly, environmental filtering dominates in less benign tropical highlands as well as across

132 most of the elevational gradient in temperate and polar regions, characterized by the strong selective

133 force of their harsher winters. This mirrors earlier findings on the increasing role of abiotic contraints

134 leading to phylogenetic clustering (32-34) and faster rates of speciation (24) at high elevations. Contrary

135 to our predictions, however, we find increasing functional overdispersion toward the highlands in

136 temperate and arctic regions. This is consistent with findings of functional overdispersion at both ends

137 of the resource availability spectrum (35). We suggest that in the already harsh conditions of higher

138 latitudes inhabited by a restricted pool of clades, higher elevations impose severe limitations where

139 facilitative interactions (35), potentially paired with competitive exclusion, becomes predominant. We

140 elucidate more of the mechanisms of community assembly by showing that local functional redundancy

141 drives the centers of functional clustering (tropical highlands and temperate low to mid elevations),

142 while overdispersion (the tropical low to mid elevations and temperate highlands) is driven 
143 predominantly by functionally unique species. The predominance of functionally unique species in

144 temperate and polar highlands is indeed consistent with increased facilitation as facilitative interactions

145 in stressful environments tend to involve primarily functionally distinct species $(5,10,11,36)$.

147 A time-calibrated species-level phylogeny allowed us to investigate the evolutionary underpinnings of

148 species coexistence. High joint phylogenetic and functional overdispersion, i.e., when assemblages are

149 comprised of distantly related species with significant trait differences, suggests that only distantly

150 related lineages can evolve sufficient niche differences to overcome competitive dynamics and attain

151 coexistence. In contrast, functional overdispersion coupled with phylogenetic clustering indicates that

152 closely related species readily evolve trait dissimilarities to avoid competition. Functional and

153 phylogenetic clustering, on the other hand, means that closely related species share adaptations that

154 allow them to persist in local environmental conditions. Finally, functional clustering in hand with

155 phylogenetic overdispersion implies that distantly related species adapt convergently to the local

156 environment. Our results suggest that the interplay of these ecological and evolutionary processes of

157 community assembly shows strong elevational and latitudinal variation.

159 Specifically, in tropical low to mid elevations, where phylogenetic overdispersion offers reasonable

160 surrogacy for functional overdispersion, distantly related species might often coexist thanks to having

161 evolved significant trait dissimilarities. As elevation in the tropics increases, phylogeny remains a

162 reasonable predictor of the functional structure, suggesting that close relatives often share trait

163 combinations conferring the ability to tolerate increasingly harsh environmental conditions of the

164 highlands. With increasing latitude, phylogeny provides a reliable proxy only for clustered assemblages,

165 again suggesting that closely related species that occur in low and mid elevations share phylogenetically

166 conserved adaptations allowing them to persist in stressful environmental conditions of temperate and 
167 polar regions. In contrast, functional overdispersion that predominantly characterizes temperate and

168 polar highlands cannot be inferred from the phylogenetic structure, implying that close relatives in the

169 highlands of high latitude regions evolve niche differences to avoid competition. These results suggest

170 that phylogeny might be a reasonable substitute for assemblage functional composition in places where

171 harsh environmental conditions constrain assemblages to a few, functionally similar species. However,

172 the relationship is inconsistent or weak for assemblages where highly functionally unique species

173 prevail. In such settings, it is ill-suited for identifying biotic constraints as a dominant assembly

174 mechanism and for recognizing regions with particularly distinct ecosystem-relevant functions.

175

176 The inconsistent predictive power of phylogeny highlights how the mechanistic interpretation of

177 assemblage functional structure across scales benefits from an assessement of its composition and

178 turnover, down to the level of single traits and their respective drivers $(29,30)$. Globally, we find a

179 strong among-assemblage trait volume redundancy from low to mid elevations and increasing

180 uniqueness above. We attribute the escalating dissimilarity in trait volumes along elevation to decreases

181 in species richness and its associated functional diversity (i.e., nestedness sensu (37)) rather than

182 turnover in actual species identities or their functions. As elevation increases, species holding functions

183 reflecting elevational, treeline or terrain, dependencies of associated habitats drop out from

184 assemblages. However, strong latitudinal variation in functional dissimilarity patterns and select

185 underlying traits exists. In the tropics, increasingly depauperate assemblages are functionally nested

186 along elevation as environmental filtering becomes predominant toward higher elevations. In temperate

187 and arctic regions, ubiquitous strong environmental filtering results in relatively stable among-

188 assemblage functional similarity along elevation. 
190 Inferring mechanisms of community assembly from observational data is admittedly challenging in the

191 absence of additional evidence from experimental manipulation $(14,38)$. Nonetheless, afforded by the

192 concurrent examination of the functional and phylogenetic structure, our analysis provides a powerful

193 test of assembly processes across scales. The additional assessement of the compositional turnover in

194 trait space paired with detailed examination of change in multiple traits along a demonstrable

195 environmental gradient (14) further allows disentangling the mechanisms that might otherwise be

196 masked by multivariate patterns (35).

198 The uncovered patterns of functional community assembly and turnover have important conservation

199 implications. At local scales, high assemblage functional uniqueness paired with low species richness

200 makes temperate and polar highlands disproportionately susceptible to the loss of critical ecological

201 functions. At larger scales, sustaining the regional multi-functionality of ecosystems requires high

202 dissimilarity among assemblages $(39,40)$ because no single, local assemblage can support all ecosystem

203 processes (41). We find such high among-assemblage functional uniqueness for tropical and sub-tropical

204 highland ecosystems, causing them to be exceedingly susceptible to disruption of their large-scale

205 functioning. Globally, different processes and scales combine to make high elevation ecosystems

206 exceptionally prone to future ecological perturbations and loss of functions.

\section{Materials and Methods}

209 Mountain systems. To delineate mountain regions, we used the inventory of the World's mountain

210 regions based on expert delineation and terrain ruggedness provided by the Global Mountain

211 Biodiversity Assessment (GMBA, http://www.mountainbiodiversity.org). The GMBA inventory identified

21246 broad-scale mountain regions across the five continents. (24) evaluated the biological independence 
213 of the GMBA delineation and found that only a small proportion of species is shared among the 46

214 regions (median similarity of $0.47 \%+-10.37 \%(s d)$ ).

215

216 Distributions and elevational ranges and biodiversity sampling. Data on breeding distributions were

217 compiled from the best available sources for a given broad geographical region or taxonomic group and

218 totaled 9993 species (for individual maps, see https://mol.org). The database of bird elevational ranges

219 was compiled by (24) and is available at https://mol.org. Using these distributional and elevational

220 ranges, we then followed the protocol established by (24) to sample biodiversity data. The sampling

221 procedure returned a total of 21,655 assemblages across the world and 8,410 assemblages within our

222 delimited mountain systems with a median of 71 per mountain region (minimum 3 in the Central

223 Australian mountains, maximum 1,816 in the Himalayas). While admittedly coarse-scale and solely

224 presence-absence, the distributional data used here comprise the best current understanding of bird

225 distributions at the global scale.

227 Avian phylogenetic and functional diversity, and trait space. For each assemblage, we calculated avian

228 dendrogram-based functional diversity (FD) (42). We based estimates of FD on a compilation of

229 function-relevant traits in (26). Four trait categories were included: body mass, nocturnality, diet, and

230 foraging niche. The diet and foraging niche categories included seven axes each: proportions of

231 invertebrates, vertebrates, carrion, fresh fruits, nectar and pollen, seeds, and other plant materials in

232 species' diet (diet category); proportional use of water below surface, water around surface, terrestrial

233 ground level, understory, mid canopy, upper canopy and aerial (foraging niche category). Following

234 existing practice $(2,43)$ we calculated multivariate trait dissimilarity using Gower's distance for each

235 pairwise combination of all 9,993 species in the dataset. Equal weights were given to each of the trait

236 categories and to each axis within the trait categories (i.e., each diet and foraging niche variable was 
237 given a $1 / 7$ weight, whereas the weights of body mass and nocturnality was 1$)$. We used Gower's

238 distance as distance metric because this index can handle quantitative, semi-quantitative, and

239 qualitative variables and assign different weights to individual traits (43).

241 The functional dendrogram was built using UPGMA (Unweighted Pair Group Method with Arithmetic

242 Mean) clustering. UPGMA clustering has the highest cophenetic correlation coefficient among most

243 popular clustering methods (Ward, Single, Complete, WPGMA, WPGMC, and UPGMC clustering

244 methods) and the lowest 2-norm index (44), ensuring most faithful preservation of the original distances

245 in the dissimilarity matrix. At each of the point locations, the functional dendrogram was pruned of the

246 branches for species that did not occur at that location to reflect that location's species composition.

$247 \quad \mathrm{FD}_{\mathrm{D}}$ was calculated as a sum of branch lengths of such local functional dendrogram.

249 The calculation of dendrogram-based phylogenetic diversity (PD) followed the same procedure (45), but

250 instead of a functional dendrogram, we used 20 dendrograms sampled from full pseudo-posterior

251 distribution of phylogenetic trees assembled by (25) (http://birdtree.org/). PD was calculated as the

252 total branch length of tree branches averaged over the 20 phylogenetic trees, with 20 trees thought to

253 provide a sufficiently strong initial estimate (46).

255 We used the same local functional dendrogram to quantify the assemblage mean (FDlavg) and skewness

256 ( FDI $\left._{\text {skew }}\right)$ of species' local functional distinctness (FDI). FDI was calculated as the fair proportion branch

257 length of the branches in a local functional dendrogram leading to a species tip and measures the

258 uniqueness of a species functional characteristics in the context of all other species in the same

259 assemblage. As such, FDI decomposes overall FD into individual species contributions. When averaged

260 across the entire assemblage, $\mathrm{FDl}_{\text {avg }}$ informs on how the average distinct functional position of single 
261 species changes along gradients ( $\left.\mathrm{FDI}_{\mathrm{avg}}\right)$. High $\mathrm{FDI}_{\text {avg }}$ indicate on average a given assemblage is comprised

262 of functionally unique species. Skewness of the distribution of FDI values further informs on whether

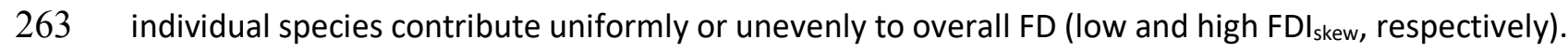

265 The clustering algorithm can affect the shape of the functional dendrogram and, consequently, the

266 estimate of the dendrogram-based functional diversity (47). To ensure that our choice of the clustering

267 algorithm did not significantly alter the inferences, we further quantified functional diversity directly

268 with the multidimensional functional space. Specifically, we calculated functional diversity as the

269 assemblage trait volume $\left(F D_{H}\right)$, quantified using the hypervolume metric (48). To obtain $F D_{H}$, we first

270 conducted the Principal Coordinate Analysis ( $\mathrm{PCOA}$ ) using the multivariate trait dissimilarity matrix based

271 on Gower's distance for each pairwise combination of all species in the dataset. We then used the first

272 two principal coordinates to quantify the hypervolume of each point location using the minimal convex

273 hull method in the package hypervolume (48). Using more than two principal coordinates was

274 computationally unfeasible because the algorithm becomes exponentially inefficient in high

275 dimensionalities. Hypervolumes quantified in this manner reflected positions of all species present at a

276 given assemblage. Results for $\mathrm{FD}_{\mathrm{H}}$ are provided in the Supplementary Material (Fig. S2).

278 To further evaluate how assemblage trait volume changes with elevation, we quantified an overlap in

279 trait volume $\left(\mathrm{FD}_{\mathrm{O}}\right)$ among the point locations falling along the elevational gradient using package

280 hypervolume (48). For each point at an elevation $a$, we selected the geographically closest point that lies

281 within $(a+500 \mathrm{~m}, a+1000 \mathrm{~m})$ elevational region. Because we wanted to evaluate changes in trait volume

282 along elevation rather than changes that might arise from geographic distance, we excluded all pairs of

283 points that were geographically farther apart than $500 \mathrm{~km}$. For each pair of points we quantified niche

284 overlap using four metrics: Sørensen (FDo) and Simpson's (FDT) similarity and unique fractions of trait 
285 volume for points at lower (FDul) and higher (FDuh) elevation (Blonder et al., 2014). To obtain these

286 indices, we first conducted the Principal Coordinate Analysis (PCoA) using the multivariate trait

287 dissimilarity matrix based on Gower's distance for each pairwise combination of all species in the

288 dataset. We then used the first two principal coordinates to quantify the hypervolume of each point

289 location using the minimal convex hull method in the package hypervolume (48). Sørensen similarity

290 was then given as twice the intersection of trait volumes of the assemblage at lower elevation and that

291 at upper elevation divided by volume of both assemblages. Unique fractions were calculated as the

292 unique component of the trait volume of, respectively, lower and higher elevation assemblage divided

293 by the entire trait volume of that assemblage. Simpson's similarity (not provided by the hypervolume

294 package) was derived from unique fractions indices and equaled to the intersection of trait volume of

295 assemblage at lower elevation and that at higher elevation divided by trait volume of the assemblages

296 with a smaller unique fraction (37). We conducted sensitivity analysis by repeating the above steps for

297 points falling within $(a+250 \mathrm{~m}, a+750 \mathrm{~m})$ and $(a+750 \mathrm{~m}, a+1250 \mathrm{~m})$, and for pairs of points located

298 geographically not farther than $100 \mathrm{~km}$ and $250 \mathrm{~km}$, and present these results in the Supplementary

299 Material (Fig. S9).

300

301 We also assessed individual components of avian trait volume. To separate trait axes, or guilds, we used

302 dietary niche (i.e., proportions of different types of food in species' diet-invertebrates, vertebrates,

303 carrion, fresh fruits, nectar and pollen, seeds, and other plant materials), foraging niche (i.e., the

304 proportional use of each of seven niches-water below surface, water around surface, terrestrial ground

305 level, understory, mid canopy, upper canopy, and aerial), nocturnality, and log-transformed body mass.

306 For dietary and foraging niche, we quantified the relative proportion (i.e., prevalence) of each diet and

307 foraging niche for each assemblage. To account for the effect of separate mountain ranges, we first 
assessed individual components of avian trait volume for each mountain range and then averaged those

309 values to obtain a global estimate.

310

311 Richness-controlled avian phylogenetic and functional diversity, and trait space. Multivariate

312 components of phylogenetic and functional diversity are often closely associated with species richness

313 (SR), and interpretations of assemblage structure benefit from statistically controlling for this

314 association. To evaluate whether elevational gradients in phylogenetic, functional, and trait diversity

315 deviate from the expectation given $\mathrm{SR}$, we generated null model for $\mathrm{PD}, \mathrm{FD}, \mathrm{FDI}_{\mathrm{avg}}, \mathrm{FDI}_{\text {skew }}, \mathrm{FD}_{\mathrm{H}}$, and

316 individual components of avian assemblage trait volume. Even though FDI values provide estimates of

317 individual species contributions to $\mathrm{FD}$, their assemblage-level means $\left(\mathrm{FDI}_{\text {avg }}\right)$ and skewness $\left(\mathrm{FDI} \mathrm{I}_{\text {skew }}\right)$ are

318 often closely associated with SR (27). Consequently, species richness-controlled equivalents of FDI ${ }_{\text {avg }}$ and

$319 \mathrm{FDI}_{\text {skew }}$ allow more in-depth interpretations of the mechanisms behind the patterns of assemblage

320 structure.

322 First, we developed the expected values of all metrics by randomly selecting species from a regional

323 species pool (i.e., species that occur in a given mountain region), keeping point-level SR constant (49).

324 The random selection of species for null models was performed 100 times. We calculated the

325 standardized effect sizes as the difference between observed values and those expected from the null

326 model, expressed in units standard deviation. The standardized effect sizes were considered the species

327 richness-controlled equivalents of biodiversity metrics and were denoted as CPD, cFD, cFDI ${ }_{\text {avg }}, \mathrm{CFDI}_{\text {skew, }}$,

328 and $\mathrm{CFD}_{\mathrm{H}}$. Negative and positive values of standardized effect sizes indicated that an observed value was

329 lower and higher, respectively, than the average expected value. Negative and positive values of cPD,

$330 \mathrm{cFD}, \mathrm{CFDI}_{\mathrm{avg}}$, and $\mathrm{CFD}_{\mathrm{H}}$ suggested clustering and overdispersion, respectively. Negative and positive 
331 values of $\mathrm{cFDI}_{\text {skew }}$ suggested less and more, respectively, even distribution of species functional

332 distinctness values.

334 Because standardized effect sizes might be affected by the shape of the null distribution, it is

335 recommended to additionally calculate quantile values (49). We thus futher calculated quantile scores

336 for the observed values of PD, FD, FDl ${ }_{\text {avg }}, F I_{\text {skew }}$, and $F D_{H}$, and estimated their associated p-values (i.e.,

337 two-tailed test, $\alpha=0.05$ ). The observed values falling outside the $2.5 \%$ and $97.5 \%$ quantiles of the null

338 distribution were considered statistically significantly lower (clustering) and higher (overdispersion),

339 respectively, than expected given SR. While the calculation of quantile scores and the associated p-

340 values removes some of the biases potentially resulting from the the shape of the null distribution, they

341 also remove information regarding the size of the effect itself (49). We thus report both the

342 standardized effect sizes (i.e., $\mathrm{CPD}, \mathrm{cFD}, \mathrm{cFDl} \mathrm{avg}_{\mathrm{av}} \mathrm{CFDI} \mathrm{skew}_{\text {, and }} \mathrm{FFD}_{\mathrm{H}}$ ) and the quantile scores with

343 associated $p$-values.

345 We then quantified frequency $(\mathrm{Fr})$ with which phylogenetic structure predicted functional structure of

346 an assemblage. Fr of successful predictions was calculated separately for overdispersed and clustered

347 assemblages and was given by the proportion of phylogenetically overdispersed (clustered) assemblages

348 that were also functionally overdispersed (clustered). We quantified Fr for both designations of

349 overdispersion and clustering: (a) all positive and negative values of standardized effect sizes were

350 considered to be overdispersed and clustered and (b) observed values outside the $2.5 \%$ and $97.5 \%$

351 quantiles of the null distribution were considered to be overdispersed and clustered, respectively. To

352 account for the effect of separate mountain ranges, Fr was averaged per mountain region, and

353 mountain region's central latitude was used to evaluate the latitudinal gradient in Fr. 
Global elevational gradients of avian functional, phylogenetic, and trait diversity. To explore how PD,

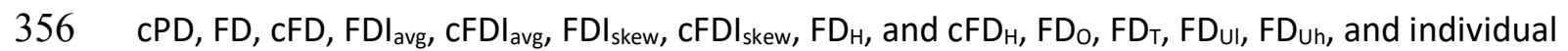

357 components of assemblage trait volume vary with elevation, we used two different models: following a

358 linear relationship,

$$
\mu_{i}=\beta_{0}+\beta_{1} x_{i} \text { (Eq. 1) }
$$

and a quadratic relationship,

$$
\mu_{i}=\beta_{0}+\beta_{1} x_{i}+\beta_{2} x_{i}^{2} \text { (Eq. 2) }
$$

367 To estimate a global pattern, we used two-level generalized hierarchical model, in which the parameters

368 for each mountain system came from a multivariate normal distribution (for model details, see (24)).

369 Log-transformed PD, FD, FDist ${ }_{\text {avg }}$, and FDist skew $_{1}, F_{H}$, as well as $c P D, c F D, c F D I_{\text {avg, }}$ and $c F D I_{\text {skew }}, \mathrm{cFD}_{H}$

370 followed a Gaussian distribution $N\left(\mu_{i}, \sigma_{i}^{2}\right) . \mathrm{FD}_{\circ}, \mathrm{FD}_{\mathrm{T}}, \mathrm{FD}_{\mathrm{UI}}, \mathrm{FD} \mathrm{Uh}$, and each component of avian trait space

371 followed a beta distribution beta $\left(\alpha_{i}, \beta_{i}\right)$; with exception of log-transformed body mass, which followed

372 a Gaussian distribution. All predictors were standardized by rescaling the distribution to have mean of 0

373 and standard deviation of 1. Each model was fitted in a Bayesian framework using INLA (50) through the

374 R-INLA package for R v.3.4.3 (https://www.r-project.org/). To select the best fitting model (quadratic or

375 linear), we estimated the Watanabe-Akaike information criterion (WAIC), which is preferable to similar

376 alternatives because it averages over the posterior distribution rather than using only point estimates

377 (51). We did not use the coefficient of determination, $R^{2}$, to evaluate goodness-of-fit of each model 
378 because $R^{2}$ does not integrate across the full posterior probability and thus does not account for

379 uncertainty in parameter estimates.

381 Latitudinal influences on elevational gradients of avian functional, phylogenetic, and trait diversity. In

382 order to explore influences of latitudinal position on the elevational gradients in PD, FD, FDistavg,

383 FDist $_{\text {skew }}, F D_{H}, c P D, c F D, c F D l_{\text {avg }}, \mathrm{CFDI}_{\text {skew }}, \mathrm{cFD}_{\mathrm{H}}, \mathrm{FD}_{\mathrm{O}}, \mathrm{FD}_{\mathrm{T}}, \mathrm{FD}_{\mathrm{Ul}}, \mathrm{FD}_{\mathrm{Uu}}$, and individual components of

384 assemblage trait volume, we expanded the global hierarchical model to include average absolute

385 latitude of the mountain system following (24). Average latitude was fitted as interaction terms with

386 linear and quadratic effects of elevation. All models were run using R-INLA. WAIC was used to select best

387 fitting model.

389 Lastly, we used the expanded best fitting global model (i.e., one including average latitude as the

390 interaction term) to predict the latitudinal gradients in PD, CPD, FD, cFD, FDI avg, $\mathrm{cFDI}_{\text {avg, }}, \mathrm{FDI}_{\text {skew, }}, \mathrm{cFDl}_{\text {skew, }}$

$391 \mathrm{FD}_{\mathrm{H}}$, and $\mathrm{CFD}_{\mathrm{H}}$ at different elevational bands.

\section{Acknowledgments}

394 The authors are grateful for support from the Translational Data Analytics Institute at the Ohio State

395 University and Yale Center for Biodiversity and Global Change. The authors also acknowledge support

396 from NSF DEB 1441737, DBI 1262600, DEB 1558568, NASA NNX11AP72G to W.J. 


\section{References}

400 1. Cardinale BJ, et al. (2012) Biodiversity loss and its impact on humanity. Nature 486:59.

$4012 . \quad$ Jarzyna MA, Jetz W (2017) A near half-century of temporal change in different facets of avian 402 diversity. Glob Change Biol 23(8):2999-3011.

403 3. Pecl GT, et al. (2017) Biodiversity redistribution under climate change: Impacts on ecosystems and 404 human well-being. Science 355(6332):eaai9214.

405 4. Peters MK, et al. (2019) Climate-land-use interactions shape tropical mountain biodiversity and ecosystem functions. Nature 568(7750):88-92.

5. Cavender-Bares J, Kozak KH, Fine PVA, Kembel SW (2009) The merging of community ecology and phylogenetic biology. Ecol Lett 12(7):693-715. Assembly through the Lens of Coexistence Theory. Annu Rev Ecol Evol Syst 43(1):227-248.

411 7. Adler PB, Fajardo A, Kleinhesselink AR, Kraft NJB (2013) Trait-based tests of coexistence

412 mechanisms. Ecol Lett 16(10):1294-1306.

413 8. Lamanna C, et al. (2014) Functional trait space and the latitudinal diversity gradient. Proc Natl Acad Sci 111(38):13745-13750.

415 9. MacArthur R, Levins R (1967) The Limiting Similarity, Convergence, and Divergence of Coexisting $416 \quad$ Species. Am Nat 101(921):377-385.

417 10. Callaway RM, et al. (2002) Positive interactions among alpine plants increase with stress. Nature 
419 11. Valiente-Banuet A, Verdú M (2007) Facilitation can increase the phylogenetic diversity of plant

$420 \quad$ communities. Ecol Lett 10(11):1029-1036.

421 12. Gallien L, Zurell D, Zimmermann NE (2018) Frequency and intensity of facilitation reveal opposing

422 patterns along a stress gradient. Ecol Evol 8(4):2171-2181.

423 13. Kraft NJB, et al. (2015) Community assembly, coexistence and the environmental filtering

424 metaphor. Funct Ecol 29(5):592-599.

425 14. Cadotte MW, Tucker CM (2017) Should Environmental Filtering be Abandoned? Trends Ecol Evol $426 \quad 32(6): 429-437$.

427 15. Schemske DW, Mittelbach GG, Cornell HV, Sobel JM, Roy K (2009) Is There a Latitudinal Gradient in 428 the Importance of Biotic Interactions? Annu Rev Ecol Evol Syst 40(1):245-269.

429 16. Mittelbach GG, Schemske DW (2015) Ecological and evolutionary perspectives on community $430 \quad$ assembly. Trends Ecol Evol 30(5):241-247.

431 17. Ricklefs RE (2008) Disintegration of the Ecological Community. Am Nat 172(6):741-750.

432 18. Belmaker J, Jetz W (2013) Spatial Scaling of Functional Structure in Bird and Mammal Assemblages. $433 \quad$ Am Nat 181(4):464-478.

434 19. Cavender-Bares J, Ackerly DD, Hobbie SE, Townsend PA (2016) Evolutionary Legacy Effects on 435 Ecosystems: Biogeographic Origins, Plant Traits, and Implications for Management in the Era of 436 Global Change. Annu Rev Ecol Evol Syst 47(1):433-462.

437 20. Cadotte MW, Davies TJ, Peres-Neto PR (2017) Why phylogenies do not always predict ecological 438 differences. Ecol Monogr 87(4):535-551. 
21. Mazel F, Mooers AO, Riva GVD, Pennell MW (2017) Conserving Phylogenetic Diversity Can Be a

$440 \quad$ Poor Strategy for Conserving Functional Diversity. Syst Biol 66(6):1019-1027.

441 22. Mazel F, et al. (2018) Prioritizing phylogenetic diversity captures functional diversity unreliably. Nat Commun 9(1):2888.

23. Körner C, et al. (2017) A global inventory of mountains for bio-geographical applications. Alp Bot 127(1):1-15.

24. Quintero I, Jetz W (2018) Global elevational diversity and diversification of birds. Nature 555:246.

25. Jetz W, Thomas GH, Joy JB, Hartmann K, Mooers AO (2012) The global diversity of birds in space and time. Nature 491:444.

26. Wilman H, et al. (2014) EltonTraits 1.0: Species-level foraging attributes of the world's birds and mammals. Ecology 95(7):2027-2027.

27. Jarzyna MA, Jetz W (2016) Detecting the Multiple Facets of Biodiversity. Trends Ecol Evol 31(7):527-538.

28. Cadotte M, Albert CH, Walker SC (2013) The ecology of differences: assessing community assembly with trait and evolutionary distances. Ecol Lett 16(10):1234-1244.

454 29. Kraft NJB, Godoy O, Levine JM (2015) Plant functional traits and the multidimensional nature of 455 species coexistence. Proc Natl Acad Sci 112(3):797-802. 
31. Blackburn TM, Gaston KJ, Loder N (1999) Geographic gradients in body size: a clarification of Bergmann's rule. Divers Distrib 5(4):165-174.

32. Graham CH, Parra JL, Rahbek C, McGuire JA (2009) Phylogenetic structure in tropical hummingbird communities. Proc Natl Acad Sci 106(Supplement 2):19673-19678.

33. Machac A, Janda M, Dunn RR, Sanders NJ (2011) Elevational gradients in phylogenetic structure of ant communities reveal the interplay of biotic and abiotic constraints on diversity. Ecography 34(3):364-371.

34. He X, Luo K, Brown C, Lin L (2018) A taxonomic, functional, and phylogenetic perspective on the community assembly of passerine birds along an elevational gradient in southwest China. Ecol Evol

35. Spasojevic MJ, Suding KN (2012) Inferring community assembly mechanisms from functional $8(5): 2712-2720$.

36. Maestre FT, Callaway RM, Valladares F, Lortie CJ (2009) Refining the stress-gradient hypothesis for competition and facilitation in plant communities. J Ecol 97(2):199-205.

37. Baselga A (2010) Partitioning the turnover and nestedness components of beta diversity. Glob Ecol Biogeogr 19(1):134-143.

38. Mayfield MM, Levine JM (2010) Opposing effects of competitive exclusion on the phylogenetic structure of communities. Ecol Lett 13(9):1085-1093. 
477 40. Mori AS, et al. (2016) Low multifunctional redundancy of soil fungal diversity at multiple scales.

$478 \quad$ Ecol Lett 19(3):249-259.

479 41. Hillebrand H, Matthiessen B (2009) Biodiversity in a complex world: consolidation and progress in

480 functional biodiversity research. Ecol Lett 12(12):1405-1419.

481 42. Petchey OL, Gaston KJ (2006) Functional diversity: back to basics and looking forward. Ecol Lett

$4829(6): 741-758$.

483 43. Pavoine S, Vallet J, Dufour A-B, Gachet S, Daniel H (2009) On the challenge of treating various

484 types of variables: application for improving the measurement of functional diversity. Oikos

$485 \quad 118(3): 391-402$.

44. Mérigot B, Durbec J-P, Gaertner J-C (2010) On goodness-of-fit measure for dendrogram-based analyses. Ecology 91(6):1850-1859.

45. Faith DP (1992) Conservation evaluation and phylogenetic diversity. Biol Conserv 61(1):1-10.

46. Schipper AM, et al. (2016) Contrasting changes in the abundance and diversity of North American bird assemblages from 1971 to 2010. Glob Change Biol 22(12):3948-3959.

47. Villéger S, Mason NWH, Mouillot D (2008) New multidimensional functional diversity indices for a multifaceted framework in functional ecology. Ecology 89(8):2290-2301.

48. Blonder B, Lamanna C, Violle C, Enquist BJ (2014) The n-dimensional hypervolume. Glob Ecol Biogeogr 23(5):595-609. 
bioRxiv preprint doi: https://doi.org/10.1101/706523: this version posted July 18,2019 . The copyright holder for this preprint (which was not certified by peer review) is the author/funder, who has granted bioRxiv a license to display the preprint in perpetuity. It is made available under aCC-BY-NC-ND 4.0 International license.

497 50. Rue H, Martino S, Chopin N (2009) Approximate Bayesian inference for latent Gaussian models by using integrated nested Laplace approximations. J R Stat Soc Ser B Stat Methodol 71(2):319-392.

499 51. Gelman A, Hwang J, Vehtari A (2014) Understanding predictive information criteria for Bayesian $500 \quad$ models. Stat Comput 24(6):997-1016. 


\section{Figures}

504 Fig. 1. The interaction of central latitude of mountain regions with elevational gradients of avian

505 functional and phylogenetic assemblage structure. Top: fitted elevational trends for dendrogram-based

506 assemblage functional diversity (FD; A), mean (FDI avg; D) and skewness (FDI skew; G) of species' local

507 functional distinctness, and dendrogram-based assemblage phylogenetic diversity (PD; J). Middle: fitted

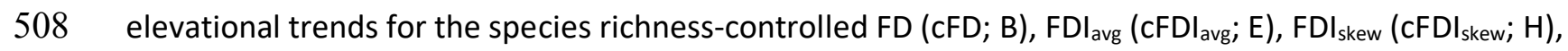

509 and PD (CPD; K), measured using standardized effect sizes. Bottom: latitudinal trends for cFD (C), cFDIavg

$510(F), c F D I_{\text {skew }}(I)$, and $c P D(L)$, given by predictions from models in (B, $E, H$, and $\left.K\right)$. Fitted global patterns,

511 with mountain ranges included as random effects in the model, are shown in dashed red line. cFD,

$512 \mathrm{cFDI}_{\text {avg }}$, and $\mathrm{cPD}$ values $>0$ and $<0$ indicate overdispersion and clustering, respectively; $\mathrm{cFDI}_{\text {skew }}$ values $>0$

513 and $<0$ indicate more and less even distribution of species FDI than expected by chance. Values of PD (in

514 MYA) were scaled by $1 / 1000$. Five highlighted in dashed line regions are the Hindukush-Himalaya (1),

515 the Andes (2), the Sumatran Islands (3), the Cameroon Mountains (4), and the Scandinavian Mountains

516 (5) ranges. Grey areas are 95\% credible intervals. For details on multi-level models see Materials and

517 Methods.

Functional structure
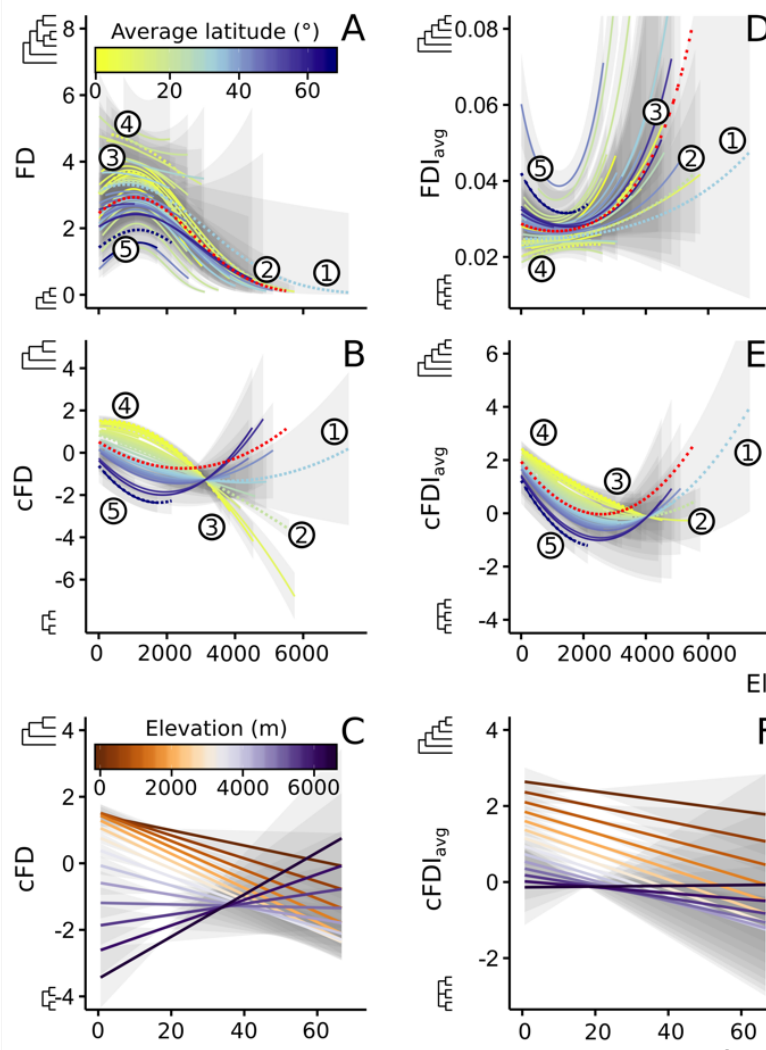


Phylogenetic structure
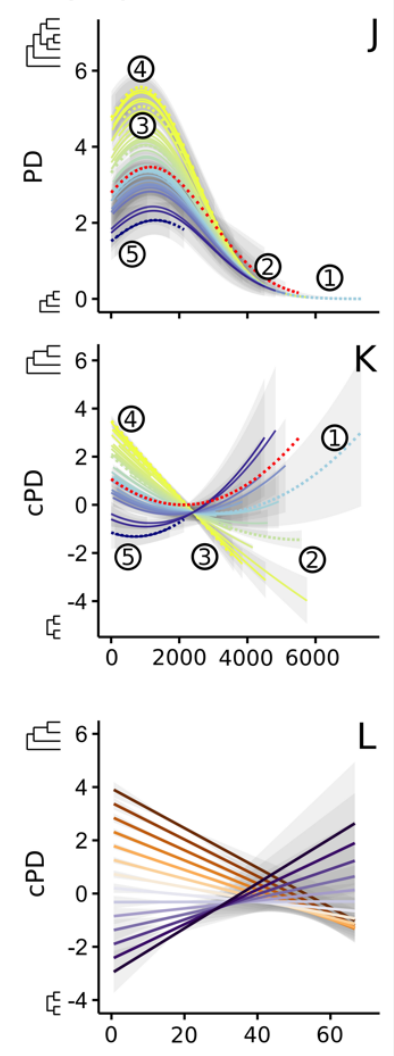
519 Fig. 2. Interaction of phylogenetic and functional structure of all 8,410 analyzed assemblages in

520 mountain regions worldwide across the latitude. Structure is given as species richness-controlled

521 phylogenetic (CPD) and functional (CFD) diversity (standardized effect sizes). Statistically significant

522 deviations in (A) and (B) are denoted by + (overdispersion), - (clustering); ns denotes non-significance;

523 numbers in the contingency table show the number of sites with a statistically significant deviation. (C)

524 illustrates the interaction of central latitude of mountain regions with the relationship between CPD and 525 cFD, with fitted aggregate global pattern shown in dashed red line. (D) illustrates the latitudinal gradient

526 of the frequency with which functional structure is accurately predicted from phylogenetic structure

527 alone. For highlighted regions see Fig. 1.

A

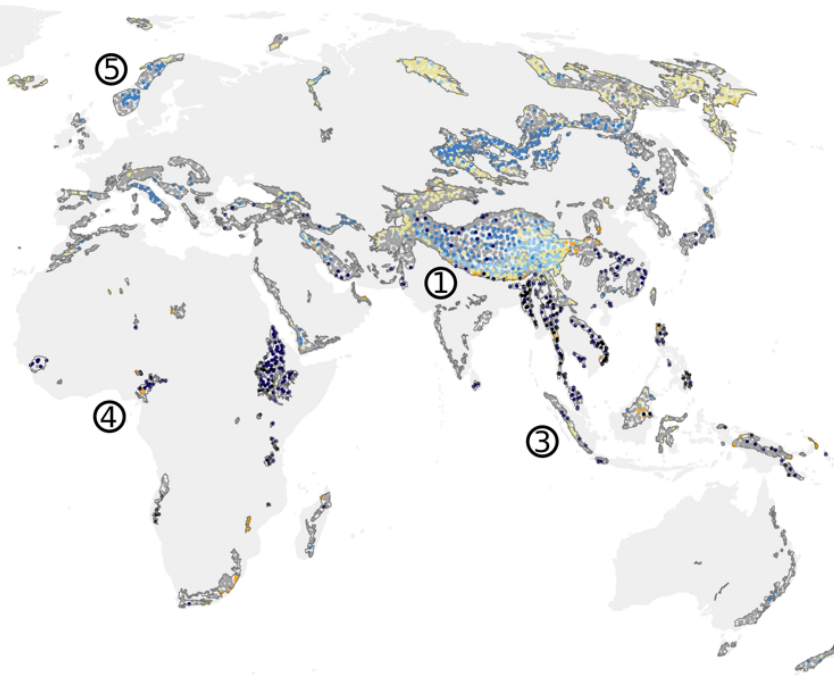

B

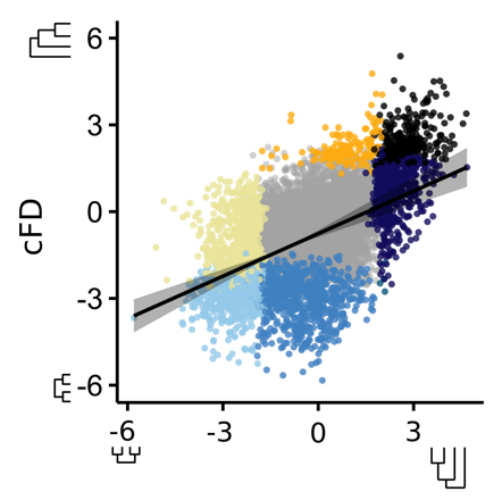

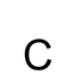

C 드 2

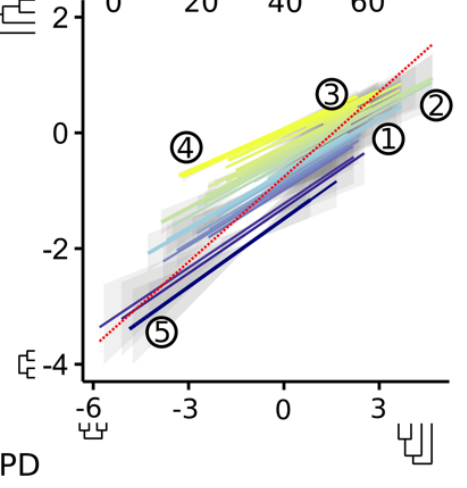

Average latitude $\left({ }^{\circ}\right)$


532 Fig. 3. Elevational gradients of assemblage trait space. (A,B) provide the two-dimensional global trait

533 space resulting from Principal Coordinate Analysis (PCOA); points are the centroids of the trait space

534 specific to each consecutive elevational band of 500m intervals averaged over all mountain regions, with

$53575 \%$ confidence ellipses encompassing assemblages at each elevational band $(A)$ and their trajectory

536 toward higher elevations (B). Size of points in (B) illustrates the number of mountain regions informing

537 each elevational band, decreasing with elevation from 42 at $500 \mathrm{~m}$ to 19 at $4,000 \mathrm{~m}$ to 1 at $6,000 \mathrm{~m}$. (C-F)

538 show the interaction of central latitude of mountain regions with elevational gradients of the overlap in

539 trait volume ( $F D_{\circ}$, Sørensen similarity; $\left.C\right)$, turnover ( $F D_{T}$, Simpson's similarity; $\left.D\right)$, and fractions of trait

540 volume unique to assemblages located at lower (FDul; E) and higher (FDuh; F) elevations. In (C-F), fitted

541 aggregate global patterns are shown in dashed red line. For highlighted regions see Fig. 1.
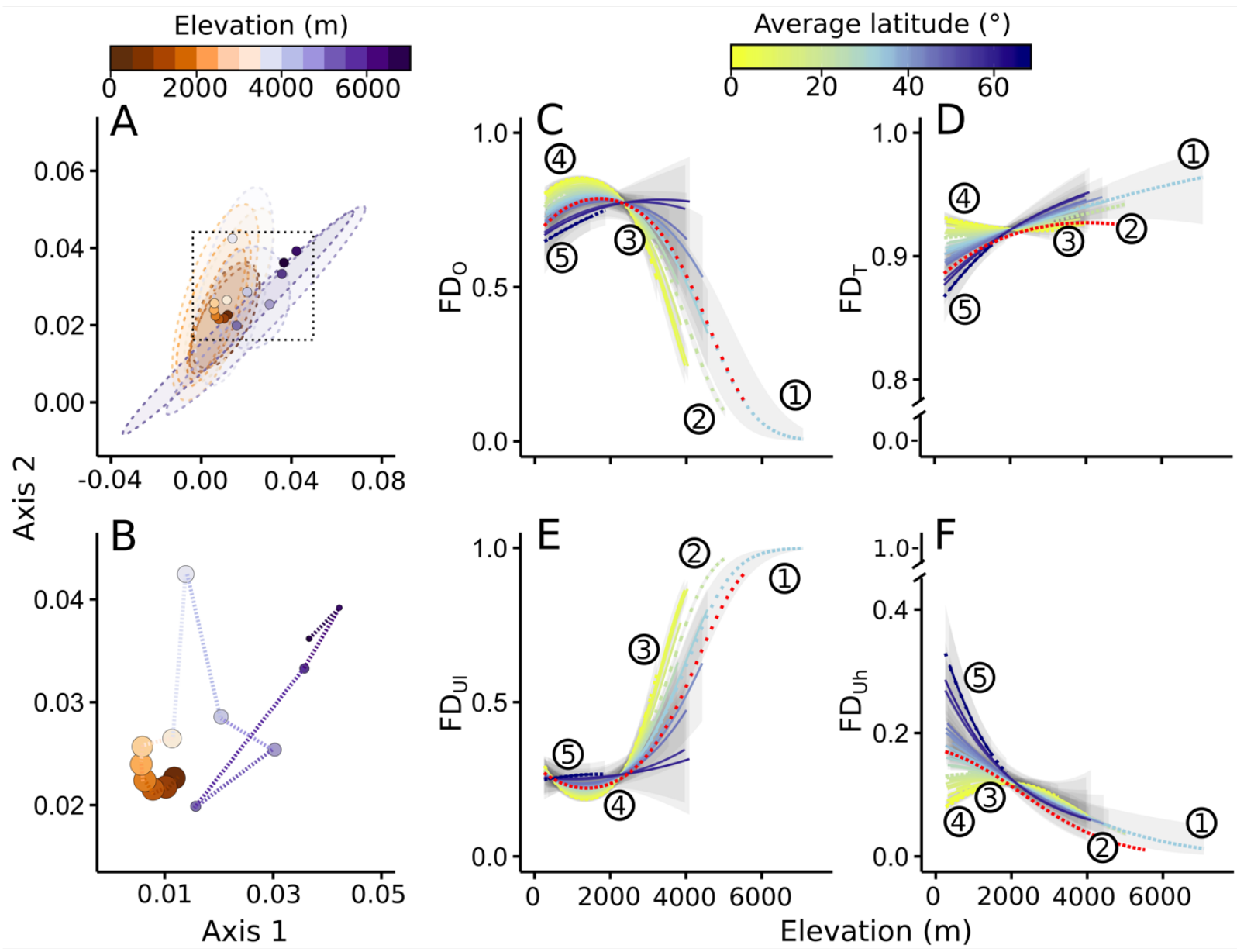
545 Fig. 4. Elevational gradients in the specific aspects of trait space. Shown are all components of the (A)

546 dietary and (B) foraging stratum axes, (C-F) selected individual traits-proportions of insectivorous diet

547 (C), ground foragers (D), nocturnality (E), and average body mass (F). (C-F) illustrate the interaction of

548 central latitude of mountain regions with elevational gradients of the given functional attribute. In (C-F),

549 fitted aggregate global patterns are shown in dashed red line. For highlighted regions see Fig. 1.

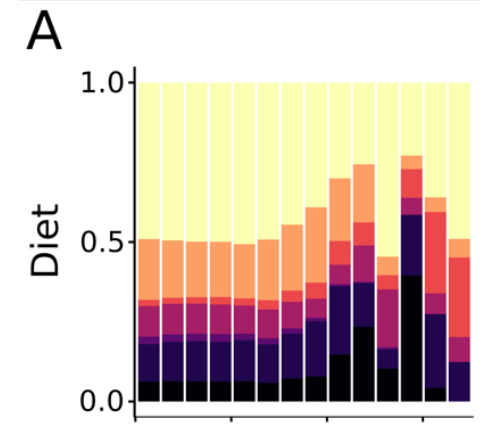

B



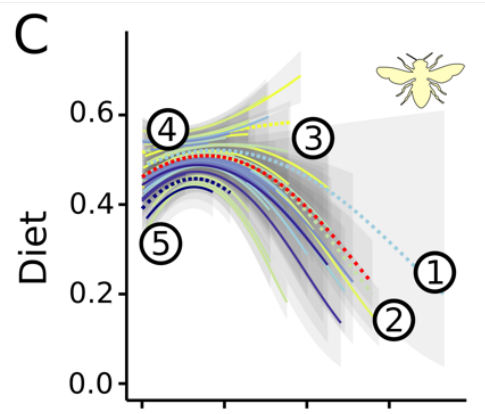

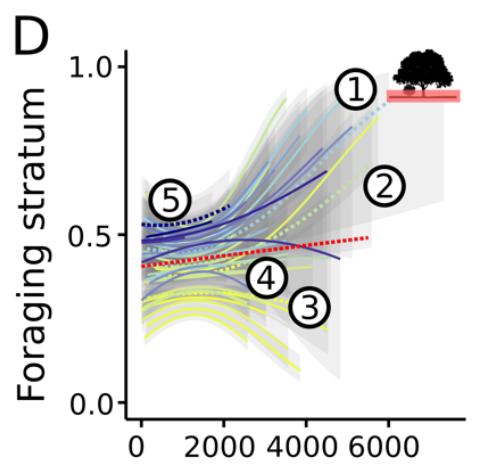

Elevation (m)

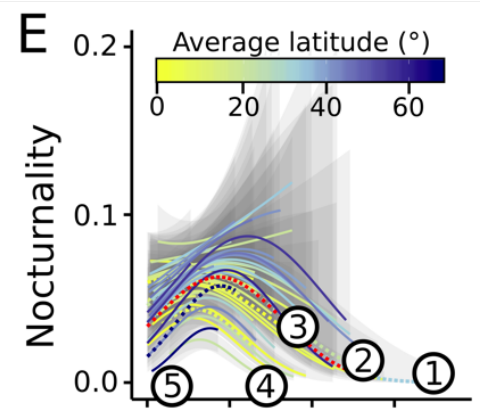

$\mathrm{F}$



